\title{
Lnk regulates integrin $\alpha$ llb $\beta 3$ outside-in signaling in mouse platelets, leading to stabilization of thrombus development in vivo
}

\author{
Hitoshi Takizawa, ${ }^{1}$ Satoshi Nishimura, ${ }^{2,3,4}$ Naoya Takayama, ${ }^{5}$ Atsushi Oda, ${ }^{6}$ Hidekazu Nishikii, ${ }^{5}$ \\ Yohei Morita, ${ }^{5}$ Sei Kakinuma, ${ }^{5}$ Satoshi Yamazaki, ${ }^{5}$ Satoshi Okamura, ${ }^{5}$ Noriko Tamura, ${ }^{7}$ \\ Shinya Goto, ${ }^{7}$ Akira Sawaguchi, ${ }^{8}$ Ichiro Manabe, ${ }^{2,3,4}$ Kiyoshi Takatsu, ${ }^{9}$ Hiromitsu Nakauchi, ${ }^{5}$ \\ Satoshi Takaki, ${ }^{1}$ and Koji Eto ${ }^{5}$
}

\begin{abstract}
${ }^{1}$ Research Institute, International Medical Center of Japan, Tokyo, Japan. ${ }^{2}$ Department of Cardiovascular Medicine, Graduate School of Medicine and ${ }^{3}$ Translational Systems Biology and Medicine Initiative, University of Tokyo, Tokyo, Japan. ${ }^{4}$ RREST, Japan Science and Technology Agency, Tokyo, Japan. ${ }^{5}$ Center for Stem Cell Biology and Regenerative Medicine, Institute of Medical Science, University of Tokyo, Tokyo, Japan.

${ }^{6}$ Department of Environmental Medicine, Graduate School of Medicine, Hokkaido University, Sapporo, Japan. ${ }^{7}$ Department of Medicine, Tokai University School of Medicine, Isehara, Japan. ${ }^{8}$ Department of Anatomy, Faculty of Medicine, University of Miyazaki, Miyazaki, Japan. 9Preferectural Institute for Pharmaceutical Research, Toyama, Japan.
\end{abstract}

\begin{abstract}
The nature of the in vivo cellular events underlying thrombus formation mediated by platelet activation remains unclear because of the absence of a modality for analysis. Lymphocyte adaptor protein (Lnk; also known as Sh2b3) is an adaptor protein that inhibits thrombopoietin-mediated signaling, and as a result, megakaryocyte and platelet counts are elevated in $\mathrm{Lnk}^{-/-}$mice. Here we describe an unanticipated role for $\mathrm{Lnk}$ in stabilizing thrombus formation and clarify the activities of Lnk in platelets transduced through integrin $\alpha I I b \beta 3$-mediated outside-in signaling. We equalized platelet counts in wild-type and $L n k^{-/-}$mice by using genetic depletion of $\mathrm{Lnk}$ and $\mathrm{BM}$ transplantation. Using $\mathrm{FeCl}_{3}$ - or laser-induced injury and in vivo imaging that enabled observation of single platelet behavior and the multiple steps in thrombus formation, we determined that Lnk is an essential contributor to the stabilization of developing thrombi within vessels. $\mathrm{Lnk}^{-/-}$platelets exhibited a reduced ability to fully spread on fibrinogen and mediate clot retraction, reduced tyrosine phosphorylation of the $\beta 3$ integrin subunit, and reduced binding of Fyn to integrin $\alpha \operatorname{IIb} \beta 3$. These results provide new insight into the mechanism of $\alpha$ IIb $\beta 3$-based outside-in signaling, which appears to be coordinated in platelets by Lnk, Fyn, and integrins. Outside-in signaling modulators could represent new therapeutic targets for the prevention of cardiovascular events.
\end{abstract}

\section{Introduction}

Platelet activation is controlled through a series of highly regulated processes and is critical for maintaining normal homeostasis (1). The nature of hemostasis and thrombosis mediated in vivo by activated platelets and its contribution to cardiovascular events remains unclear, however. Particularly challenging has been the characterization of the multicellular network of interactions among platelets, endothelial cells, leukocytes, and erythrocytes that occur during thrombus formation in pathological settings and analysis of the kinetics of platelet activity. Injury to vascular endothelial cells exposes matrix proteins, which induce platelets to adhere to the vessel wall, where they subsequently spread and become activated. At the high shear rates found within the circulation, vWF immobilized on the vessel wall binds to the platelet receptor glycoprotein Ib-V-IX complex (GPIb-V-IX), facilitating platelet adhesion to injured sites, where collagen and/or laminin are exposed $(2,3)$. Once activated, the adhering platelets secrete soluble mediators to recruit additional circulating platelets, and, through their aggregation, bleeding is stopped. Platelet activation is mediated via several signaling pathways, including the integrin

Authorship note: Hitoshi Takizawa and Satoshi Nishimura contributed equally to this work.

Conflict of interest: The authors have declared that no conflict of interest exists. Citation for this article: J. Clin. Invest. 120:179-190 (2010). doi:10.1172/JCI39503. $\alpha \operatorname{IIb} \beta 3$ pathway (1). Other receptor-ligand interactions, including the binding of GPVI-collagen, $\mathrm{P} 2 \mathrm{Y}_{1} / \mathrm{P} 2 \mathrm{Y}_{12}$-ADP, and protease-activated $G$ protein-coupled receptor-thrombin (PAR-thrombin), synergistically promote integrin $\alpha \mathrm{IIb} \beta 3$ activation (inside-out signaling) and the subsequent binding of fibrinogen or vWF to integrin $\alpha \operatorname{IIb} \beta 3$. This binding triggers signaling that promotes cytoskeletal changes that lead to the spread and stabilization of platelet thrombi through a process termed outside-in signaling $(1,2)$.

It is also known that $\alpha \mathrm{IIb} \beta 3$ physically interacts with non-receptor tyrosine kinases such as Src and Syk $(4,5)$ and that activation of these kinases upon engagement of integrin with fibrinogen contributes to the stability of thrombi in vivo (6). The kinases and adaptors involved in the assembly of the $\alpha \operatorname{IIb} \beta 3$-based signaling complex are believed to include Syk, lymphocyte cytosolic protein 2 (Lcp2, also known as SH2 domain-containing leukocyte protein of $76 \mathrm{kDa}$ [SLP-76]), Vav, and Fyn-binding protein (Fyb, also known as adhesion and degranulation promoting adaptor protein $[\mathrm{ADAP}])(4,5)$. Tyrosine phosphorylation of the cytoplasmic domain of the integrin $\beta 3$ subunit, at least on Tyr 747 , is required for stable platelet aggregation and the interaction of myosin with the $\beta 3$ subunit in platelets (7), which is believed to be necessary for full clot retraction $(8-10)$.

Lnk (SH2B adaptor protein 3 [Sh2b3]) belongs to the Src-homology 2 (SH2) adaptor family, which also includes SH2-B (Sh2b1) and adaptor protein with PH and SH2 domains (APS; Sh2b2) (11). 
A
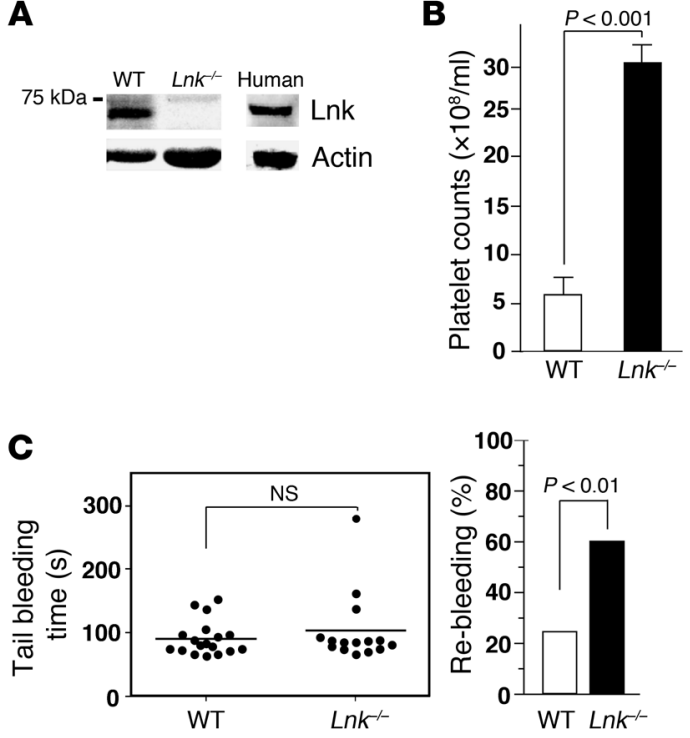

Lnk-deficient ( $\left.L n k^{-/-}\right)$mouse strains exhibit excessive accumulation of c-Kit ${ }^{+}$Sca- $1^{+}$lineage ${ }^{-}(\mathrm{KSL}) \mathrm{CD} 34^{-/ \mathrm{lo}} \mathrm{HSCs}$, B cell precursors, erythroblasts, megakaryocytes, and platelets but do not exhibit thrombogenesis and have normal longevity (11-14). The observed phenotypes of $\mathrm{Lnk}^{-/-}$mice are caused by a loss of negative regulation by Lnk signaling transduced through several growth factor and cytokine receptors, including the stem cell factor receptor $\mathrm{c}$-Kit, the erythropoietin receptor, and the thrombopoietin receptor c-Mpl $(11,12,14-17)$. In the present study, we used an $\mathrm{FeCl}_{3}$ induced injury model and our high-resolution imaging system, which enables observation of single platelet behavior in vivo (18), to examine the function of Lnk in platelets with the aim of clarifying its role in thrombosis. Our results suggest that Lnk promotes stabilization of the developed thrombus, mainly through integrin $\alpha \operatorname{IIb} \beta 3$-mediated actin cytoskeletal reorganization, and suggest this molecule may represent a new therapeutic target for the treatment and/or prevention of cardiovascular disease.

\section{Results}

Thrombus stability is impaired in Lnk L $^{-/}$mice in different mouse models of thrombosis. Lnk is expressed by megakaryocytes, where it acts through integrin signaling to regulate their growth and maturation $(14,19)$. To determine whether platelets retain Lnk after their release from megakaryocytes and, if so, what its function is, we first used immunoblotting to assess Lnk levels in platelets obtained from WT C57BL/6 mice. This analysis confirmed that substantial amounts of Lnk are retained by WT platelets, and similar results were obtained in humans (Figure 1A). Accordingly, we next investigated the function of Lnk in platelets by examining the effect of Lnk deficiency. As reported previously $(14,19)$, $\mathrm{Lnk}^{-/-}$mice showed a 5 -fold increase in platelet number (Figure 1B), though flow cytometry revealed platelet size to be unaffected by the absence of Lnk (data not shown). Moreover, transmission electron microscopic examination revealed that the intracellular structures of resting WT and $L n k^{-/}$platelets, including the $\alpha$ - and dense granules, were indistinguishable (Supplemental Figure 1A; supplemental material available online with this article; doi:10.1172/JCI39503DS1). Likewise, the expression levels of the major integrin subunits, $\alpha I I b, \alpha 2, \beta 3$, and $\beta 1$, as well as GPIb $\alpha$ (the

\section{Figure 1}

Increased numbers of platelets circulate in $\mathrm{Lnk}^{-1-}$ mice, but re-bleeding events are increased, while bleeding times are comparable. (A) Lnk levels in platelets. Washed platelets from WT and $L n k^{-1-}$ mice or human platelets were lysed and were subjected to immunoblotting with anti-Lnk and anti-actin Abs. (B) Platelet counts in EDTA-treated peripheral blood (mean \pm SD, $n=15$ each). (C) Tails of WT $(n=18)$ and $L n k^{-/-}$mice $(n=15)$ were warmed and then transected, immersed in PBS at $37^{\circ} \mathrm{C}$, and then monitored for 60 seconds so that any rebleeding would be detected (detection was positive or negative). Horizontal bars in the left panel show mean in each group.
vWF receptor) and GPVI (the collagen receptor), were also similar in WT and $L n k^{-/-}$platelets (Supplemental Figure $1 \mathrm{~B}$ ).

To examine the functional consequences of Lnk deficiency in mice, we inflicted tail wounds on the mice, after which $L n k^{-/-}$mice exhibited bleeding times that were comparable to those in WT mice. But whereas $23 \%$ of WT mice showed re-bleeding, $60 \%$ of Lnk ${ }^{-/-}$mice re-bled $\left(P<0.01\right.$ in a $\chi^{2}$ test, Figure $1 \mathrm{C}$; re-bleeding times did not differ significantly: WT, $58 \pm 8$ seconds vs. Lnk ${ }^{-/-}$, $62 \pm 6$ seconds), suggesting that thrombi formed in $L n k^{-/-}$mice are more fragile than those formed in WT mice $(1,8,20)$.

To accurately interpret the results summarized above, 2 characteristics of the system had to be taken into account: (a) the numbers of circulating platelets in $L n k^{-/-}$mice were 5-fold higher than in WT mice (Figure 1B); and (b) Lnk is expressed in endothelial cells as well as platelets (21). In order to exclude the influence of endothelial cells and platelet number, we performed BM transplantation using WT or Lnk WM $^{-/}$BM cells. Because it is well established that Lnk deficiency increases stem cell number and enhances the engraftment efficiency upon transplantation (22), we transplanted $1 \times 10^{7} \mathrm{BM}$ cells from Ly5.1 WT mice or $2 \times 10^{5}$ or $5 \times 10^{5} \mathrm{BM}$ cells from Ly5.1 $\mathrm{Lnk}^{-/-}$mice into irradiated 8-week-old Ly5.2 recipient mice, which are hereafter referred to as WT-chimeras and Lnk-chimeras, respectively (Figure 2A). We confirmed that with successful BM replacement (all mice used showed greater than 98\% chimerism at Ly5.1/Ly5.2 on myeloid-lineage cells) and with platelets lacking Lnk expression from Lnk-chimeras, the platelet counts in 16-week-old WT-chimeras (8 weeks after transplantation) were comparable to those in 12-week-old Lnk-chimeras (4 weeks after transplantation). By 8 weeks after transplantation, the Lnk-chimeras showed higher platelet counts (Figure 2B). Correspondingly, when compared with WT-chimeras at 8 weeks after transplantation, bleeding times were significantly prolonged in Lnk-chimeras at 4 weeks but not at 8 weeks $(P<0.01$, Figure $2 \mathrm{~B})$. WT-chimeras at 8 weeks, Lnk-chimeras at 4 weeks, and Lnk-chimeras at 8 weeks showed re-bleeding times of 59 seconds ( 3 of 14 mice), 246 seconds (7 of 14 mice), and 57 seconds ( 5 of 11 mice), respectively. This suggests that the Lnk deficiency itself contributes to the increased tail bleeding and re-bleeding times. Although there was a high inverse correlation between bleeding times and platelet counts in both 
A
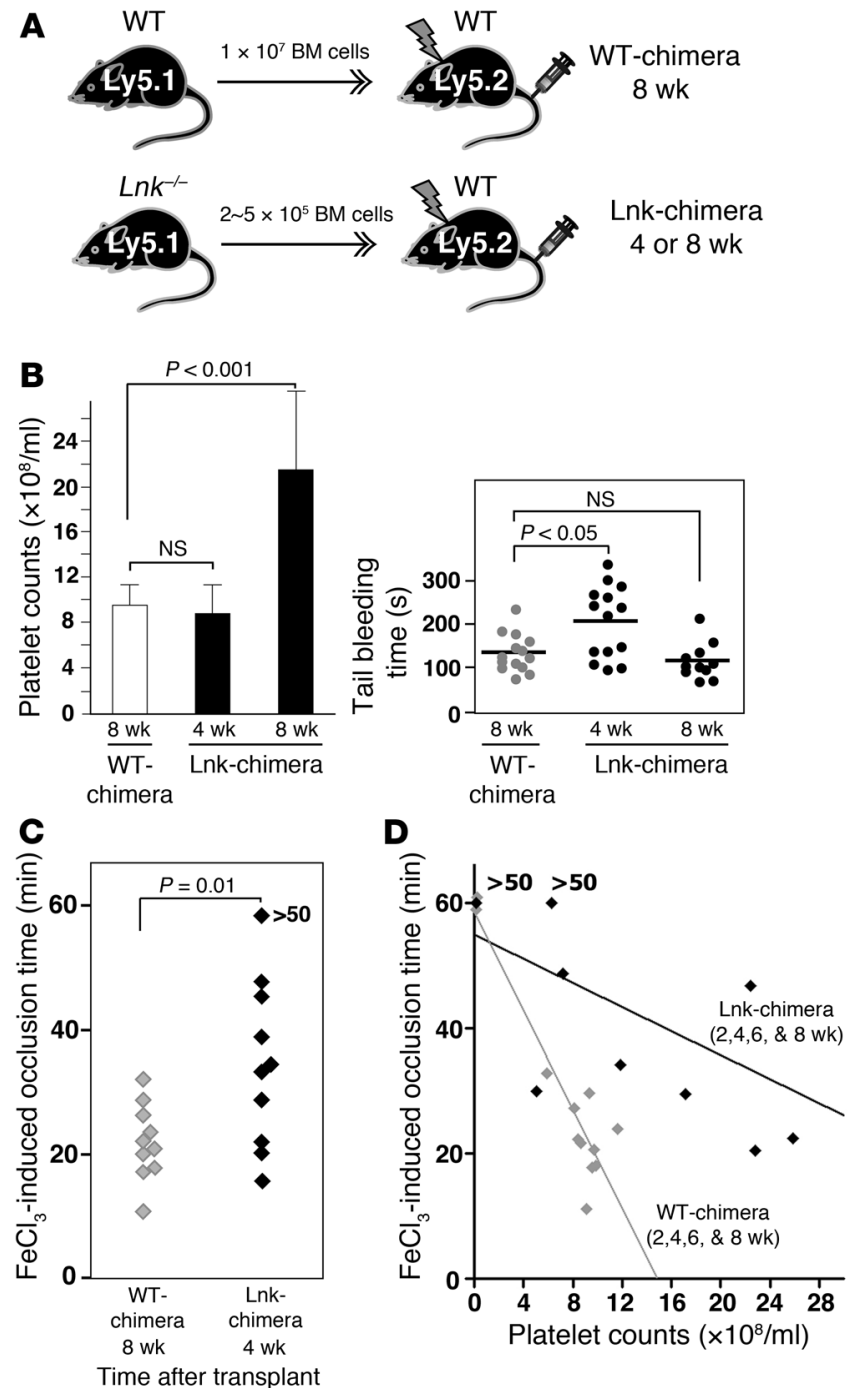

WT- and Lnk-chimeras, irrespective of platelet age or circulating platelet counts (Supplemental Figure 2), the results again suggest that the impaired thrombosis reflects the Lnk deficiency.

We then evaluated occlusion times in carotid arteries exposed to $\mathrm{FeCl}_{3}(10 \%$ solution on the adventitial side) to induce endothelial injury for assessing thrombus formation in vivo (23). We found that in WT- and Lnk-chimeras with comparable platelet counts, occlusion times were significantly longer in the Lnk-chimeras, which is also indicative of a functional defect in $L n k^{-/}$platelets (WT-chimera, $n=10$; Lnk-chimera, $n=10 ; P=0.01$, Figure 2C). When we analyzed for the relationship between occlusion times and platelet counts in the chimeras $2,4,6$, and 8 weeks after transplantation, there was a high inverse correlation between occlusion times and platelet counts. Of note, however, Lnk-chimeras exhibited longer occlusion times than WT-chimeras with similar platelet counts (Figure 2D), further confirming that Lnk deficiency impairs thrombus formation.

To assess the functionality of $L n k^{-/-}$platelets in more detail, we used a direct visual technique that enabled us to evaluate in vivo thrombus stability with much greater temporal and spatial resolution and to characterize the kinetics of $L n k^{-/-}$platelet activity involved in thrombus formation. This method is based on con-

\section{Figure 2}

Adjustment of platelet counts through BM transplantation showed prolonged bleeding and occlusion times in a $\mathrm{FeCl}_{3}$-induced thrombosis model. (A) The protocol for BM transplantation. (B) The left panel shows the platelet counts after transplantation $(n=11-14)$; data represent mean \pm SD. The right panel shows the duration of tail bleeding at the indicated time points; horizontal bars show mean in each group. (C) Platelet counts in BM-transplanted mice. WT-chimeras at 8 weeks and Lnk-chimeras at 4 weeks after transplantation were utilized to measure occlusion times during $\mathrm{FeCl}_{3}$-induced thrombosis in carotid arteries (WT-chimera, $n=10$; Lnk-chimera, $n=10 ; P=0.01$ ). (D) Relationship between occlusion times with $\mathrm{FeCl}_{3}$-induced thrombosis and platelet counts in WT- and Lnk-chimeras measured 2, 4, 6, and 8 weeks after transplantation ( $n=12$ animals in each group). Black symbols denote Lnk-chimeras, while gray ones denote WT-chimeras; the black $(y=-0.96 x+55.0)$ and gray lines $(y=-3.94 x+58.6)$ are fitted to the data from the Lnk- and WT-chimeras, respectively.

focal laser microscopy and permits high spatiotemporal resolution of individual platelets under flow conditions in mesenteric capillaries and arterioles (24). With this system, laser irradiation produces ROS, which cause injury to the endothelial layer of the vessels (18). Because laser-induced thrombosis reportedly differs from the $\mathrm{FeCl}_{3}$-induced injury model in terms of the mechanism of thrombus formation $(25,26)$, we again used WT-chimeras 8 weeks after transplantation and Lnk-chimeras 4 weeks after transplantation, as we did in the experiments shown in Figure 2C. We found that WT- and Lnk-chimeras showed similar single platelet kinetics in the absence of injury, including transient interactions with the endothelium (data not shown). After laser-induced injury, however, thrombus formation was severely diminished in the Lnk-chimeras.

After laser-induced injury to mesenteric capillaries, platelets adhered to the vessel walls at similar rates in the WT- and Lnkchimeras (WT-chimeras, $1.59 \pm 0.18$ platelets $/ \mathrm{ms} / \mu \mathrm{m}$ vs. Lnk-chimeras, $1.48 \pm 0.19$ platelets $/ \mathrm{ms} / \mu \mathrm{m} ; n=30$ vessels from 5 animals, $P=0.44$ ). In the WT-chimeras, the adherent platelets caused platelets in the flowing blood to acutely pile up, and the resultant thrombus reduced the vessel lumen diameter and blood flow velocity. Ultimately, the blood vessel was completely occluded by plugged erythrocytes and/or leukocytes. By contrast, in the Lnk-chimeras, platelets adhered to the vessel wall more loosely than in WT-chimeras, so that they were frequently washed away by the blood flow. As a consequence, the number of platelets that piled up and the size of the resultant thrombus were smaller than in WT-chimeras (Figure $3 \mathrm{~A}$ and Supplemental Videos 1 and 2). More intriguingly, in both Lnk- and WT-chimeras 2, 4, 6, and 8 weeks after transplantation, the number of platelets adhering to the vessel walls during thrombus formation appeared to be well correlated with the circulating platelet count. However, when we compared thrombus formation in Lnk- and WT-chimeras with similar platelet counts, there was a tendency for animals with $\mathrm{Lnk}^{-/-}$platelets (Lnk-chimeras) to show impaired thrombus formation, as compared with WT-chimeras. Moreover, the greatly increased platelet numbers in $\mathrm{Lnk}^{-/-}$animals did not enhance thrombus formation to levels similar to those seen in WT-chimeras (Figure 3B), which suggests that Lnk deficiency is itself a factor that contributes to the impaired stabilization of developing thrombi in our laser-induced injury model.

We also applied the laser-induced injury model to compare thrombosis in mesenteric capillaries and arterioles of 12-week-old 
A
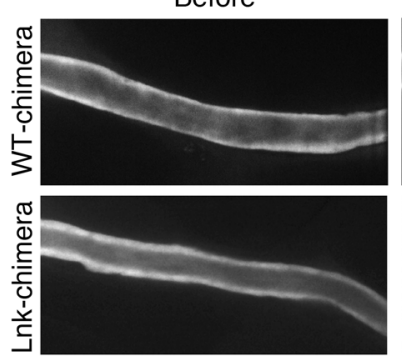

Flow $\longrightarrow$
After
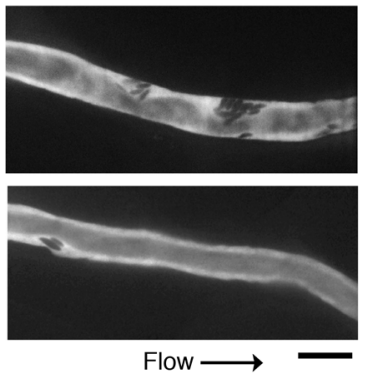

Flow $\longrightarrow \longrightarrow$

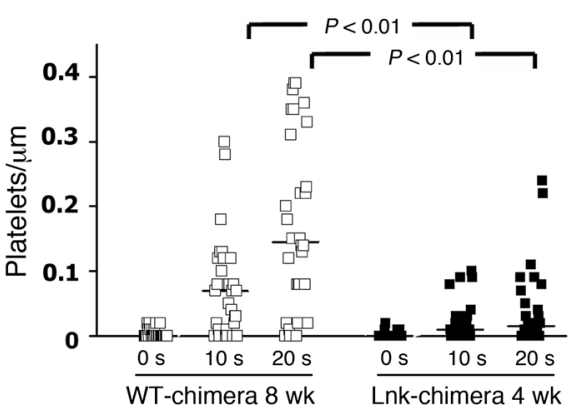

C

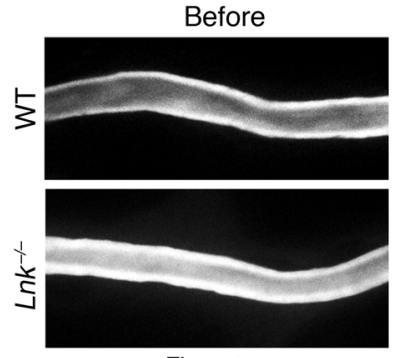

Flow $\rightarrow$
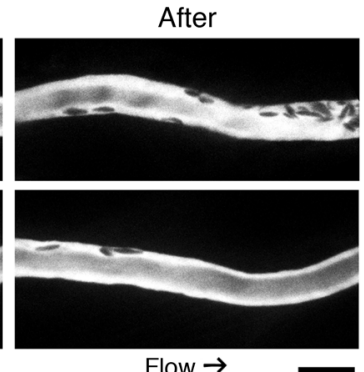

Flow $\rightarrow$

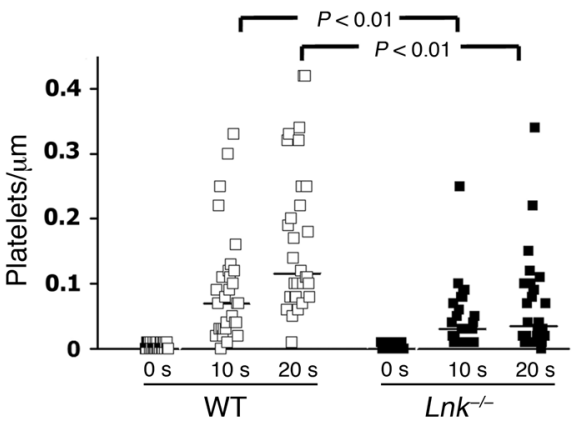

B

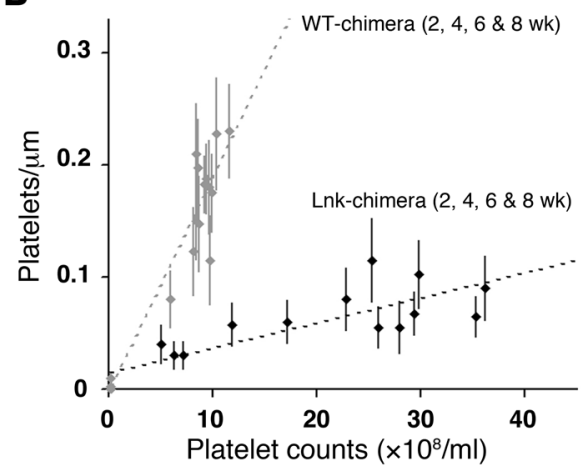

D

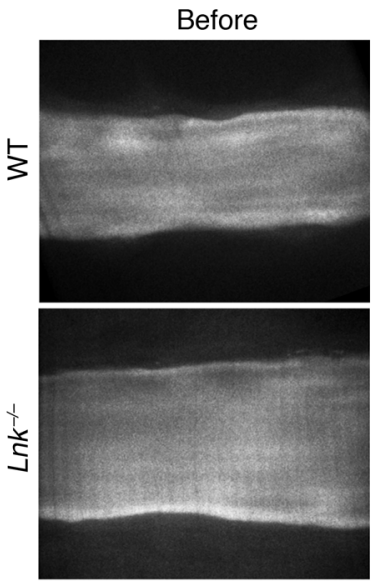

Flow $\rightarrow$

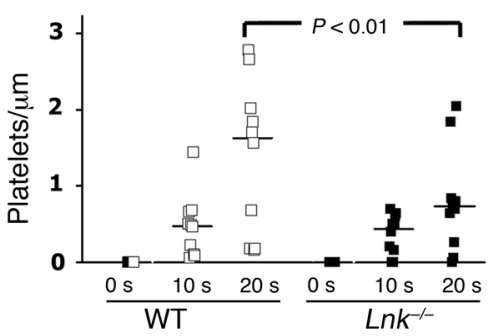

Figure 3

In vivo thrombus formation was impaired in Lnk-chimeras and $L n k^{-1-}$ mice in a laser-induced injury model. (A, C, and D) Video stills of mesenteric capillaries (A and $\mathbf{C}$ ) and arterioles (D) obtained using intravital fluorescence microscopy before and 20 seconds after laser-induced injury. The numbers of platelets in developing thrombi after laser injury to capillaries (A and $\mathbf{C}$, lower panel) and arterioles (D, lower panel) were calculated. In A, C, and D, $y$ axes represent the numbers of platelets per micrometer of observed vessel length. In A, results from WT-chimeras 8 weeks after transplantation (16 weeks old) and Lnk-chimeras 4 weeks after transplantation (12 weeks old) are shown ( $n=5$ each). (B) Relationship between platelet counts and laser-induced thrombosis. All recipient mice were studied 2, 4, 6, or 8 weeks after transplantation $(n=17$ animals for each groups). For each mouse, the numbers of platelets per micrometer contributing to thrombi after 20-second injuries to 10 mesenteric capillaries are shown as mean \pm SEM along with platelet count. Black and gray dotted lines are fitted to the data from the Lnk- and WT-chimeras, respectively. (C and D) Results from 12-week-old WT and $L n k^{-1-}$ mice $(n=5$ each). See Supplemental Videos 1-6 for original movies. Note the impaired thrombus formation in $\mathrm{Lnk}^{-1-}$ mice in both capillaries and arterioles. Scale bars: $10 \mu \mathrm{m}$. Horizontal lines indicate the median values in $\mathbf{A}, \mathbf{C}$, and $\mathbf{D}$.

WT and $L n k^{-/-}$mice (Figure 3, C and D, for capillaries and arterioles, respectively). Upon laser-induced injury, the platelet kinetics in $\mathrm{Lnk}^{-/-}$mice were similar to those seen in the Lnk-chimeric mice 4 weeks after transplantation. Initial attachment of the platelets to the vessel wall was observed; however, stable thrombus formation, including the piling up of platelets, was diminished in manner similar to that seen in the Lnk-chimeras. As a result, the numbers of platelets making up the thrombi were significantly reduced in 

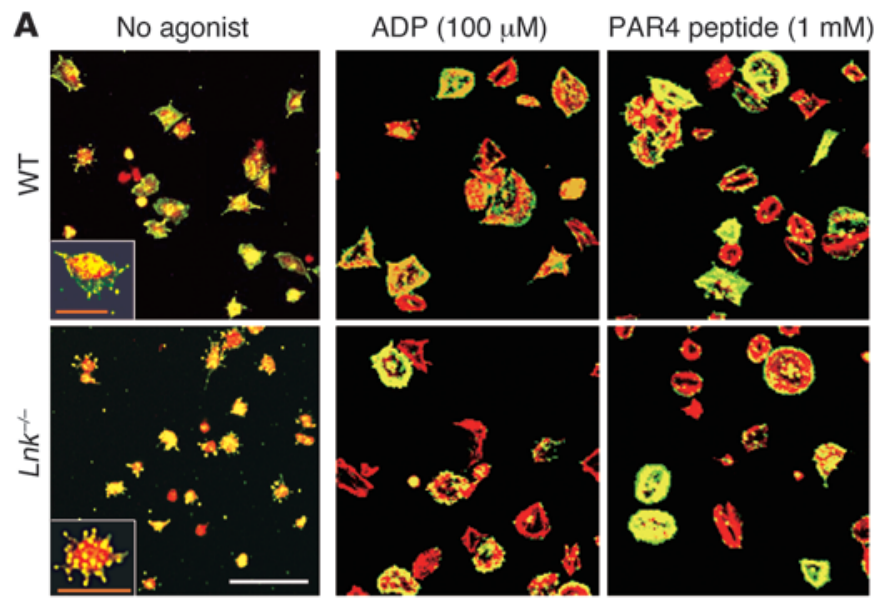

B

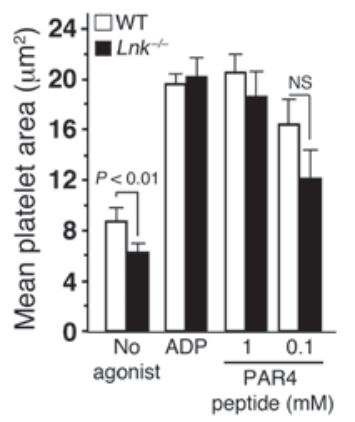

C

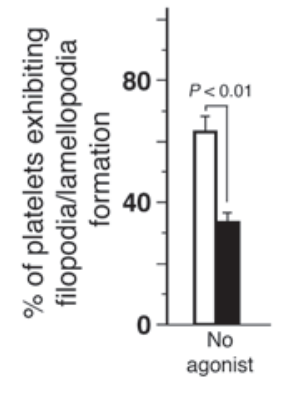

D

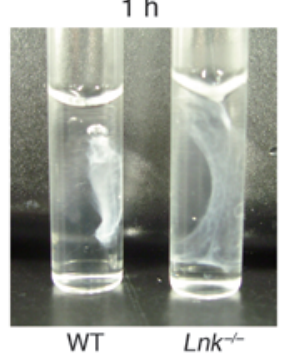

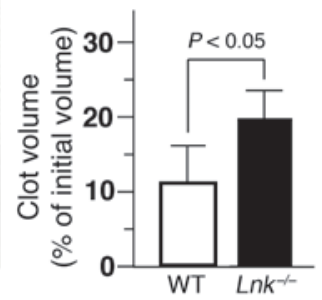

E
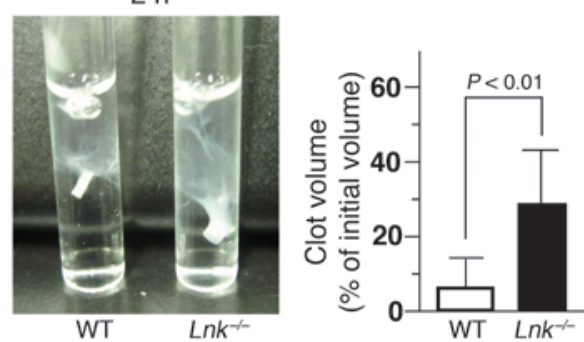

\section{Figure 4}

$L_{n k^{-1}}$ platelets show defective $\alpha$ llb $\beta 3$-dependent spreading on fibrinogen and fibrin clot retraction. (A) Washed platelets from WT and $L n k^{-1-}$ mice were plated on fibrinogen-coated coverslips for 45 minutes. In some preparations, $100 \mu$ M ADP or 0.1 or 1 mM PAR 4 receptor-activating peptide was present. Cells were fixed, permeabilized, and stained with rhodamine-phalloidin to visualize F-actin (red) and with anti-phosphotyrosine mAb (green). Scale bars: $10 \mu \mathrm{m}$ (white); $4 \mu \mathrm{m}$ (insets, orange). (B) Platelet spreading was quantified by computer analysis of their surface areas. Each bar in B represents the value (mean \pm SD) from at least 250 platelets. (C) Percentages of platelets exhibiting filopodia or lamellipodia. Twenty sections (20-30 cells/section) were analyzed, and mean \pm SD is shown. (D and E) Fibrin clot retraction was assessed at 1 and 2 hours after addition of thrombin, fibrinogen, and $\mathrm{CaCl}_{2}$ to washed platelets from WT or $\mathrm{Lnk}^{-1-}$ mice. The images show representative results at 1 (D) and 2 hours (E). The graphs show the summarized results at 1 (D) and 2 hours (E) (mean \pm SD, $n=10$ each).

both mesenteric capillaries (Figure 3C and Supplemental Videos 3 and 4) and arterioles (Figure 3D and Supplemental Videos 5 and 6). Taken together, the results indicate that the observed phenotype for thrombus formation caused by the Lnk deficiency is attributable to platelet function, per se, and does not reflect changes in endothelial cell function. This is noteworthy, as it suggests that there could be less thrombus formation in the Lnk-chimeras than in $\mathrm{Lnk}^{-/-}$mice, as the higher platelet counts in the latter might slightly compensate for their diminished functionality (Figure 3, A-C).

Lnk promotes integrin $\alpha I I b \beta 3$-mediated actin cytoskeletal reorganization but not agonist-dependent integrin activation. To determine the basis for the instability of thrombi formed by $L n k^{-/-}$platelets, we examined their ability to adhere to fibrinogen-coated plates and their morphology after spreading, both of which are dependent on outside-in $\alpha \operatorname{IIb} \beta 3$ signaling $(1,8,27)$. The initial adhesion of $\mathrm{Lnk}^{-/-}$platelets to fibrinogen-coated plates in the absence of agonistic stimuli appeared normal, as did formation of filopodial projections (data not shown). On the other hand, their subsequent spreading (after 15 minutes) was impaired, and formation of a lamellipodial edge was incomplete (shown at 45 minutes, Figure $4 \mathrm{~A}$, "No agonist") $(28,29)$. The mean area covered by the adhering platelets and the percentage of platelets showing filopodia and/ or lamellipodia (percentage of spreading) were both significantly reduced in the absence of Lnk (at 45 minutes, Figure 4, B and C; time-dependent change in mean area without agonist, Supplemental Figure 3A). This suboptimal spreading of $L n k^{-/-}$platelets was not observed when they were stimulated with a high concentration of a G protein-coupled receptor agonist such as $100 \mu \mathrm{M}$ ADP or $1 \mathrm{mM}$ PAR4-activating peptide (sequence: AYPGKF) (Figure 4, A and $B$ ), although lower concentrations of agonists, such as $0.1 \mathrm{mM}$ PAR4-activating peptide (Figure 4B) or $0.05 \mathrm{U} / \mathrm{ml}$ thrombin (Supplemental Figure 3B), did not completely restore the spreading on immobilized fibrinogen impaired by Lnk deficiency, even when the secretion of effectors from platelet granules was blocked (Supplemental Figure 3B). In addition, there was no significant difference in the reduction in the spread areas of platelets from Lnk-chimeras 4 weeks and 8 weeks after transplantation (data not shown), indicating that the impaired spreading caused by Lnk deficiency is independent of platelet age after myelosuppression. Thus, Lnk appears to continuously participate in a subset of $\alpha \operatorname{IIb} \beta 3$ - and actin-dependent morphological responses triggered by platelet adhesion to fibrinogen (1) independently of its negative impact on proliferation in HSCs and megakaryocytes $(12,14,19)$.

Another platelet response that is dependent on $\alpha \operatorname{IIb} \beta 3$ and the actin cytoskeleton is fibrin clot retraction $(8,9,27)$, which we examined using equal numbers of $L n k^{-/-}$and WT washed platelets 
A

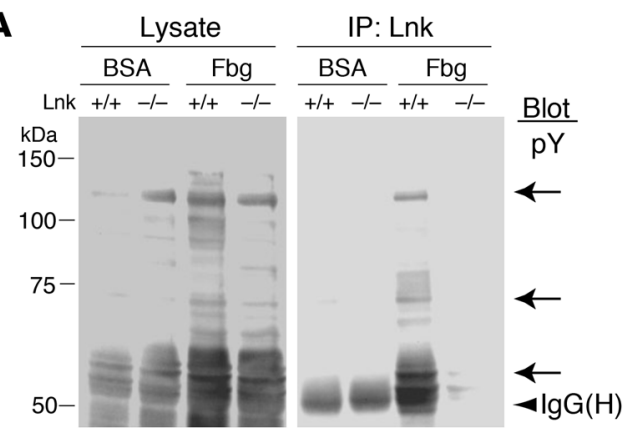

C

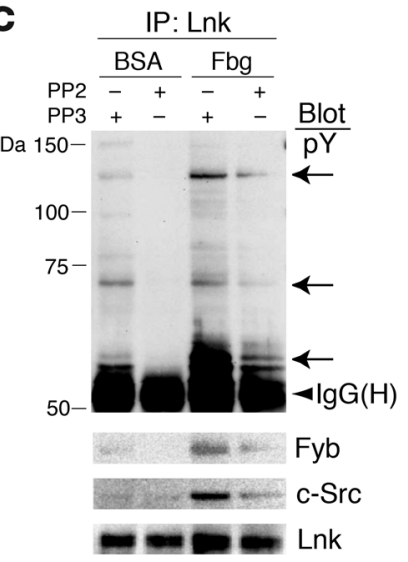

B

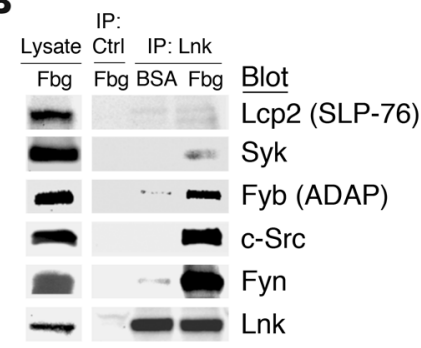

Figure 5

Lnk associates with c-Src, Fyn, and Fyb in a manner dependent on outside-in signaling. (A) Washed platelets from WT and $L n K^{-1-}$ mice were plated on fibrinogen ( $\mathrm{Fbg}$ ) for $45 \mathrm{~min}$ utes or maintained in suspension in a BSAcoated dish. Platelet lysate (left) or proteins immunoprecipitated using anti-Lnk Abs (right, IP: Lnk) were separated and probed with anti-phosphotyrosine (pY) mAb. (B) Lysates from WT platelets prepared as in $\mathbf{A}$ and immunoprecipitates obtained using irrelevant control rabbit sera (IP: Ctrl) or anti-Lnk (IP: Lnk) were probed by immunoblotting for the indicated proteins. (C) WT platelets were incubated with $5 \mu \mathrm{M}$ PP2 to block Src kinase activity or with PP3, an inactive congener of PP2. PP2 but not PP3 diminished tyrosine phosphorylation of cellular proteins and the association of c-Src and Fyb, with Lnk in platelets adhering to fibrinogen. The observation was confirmed using $20 \mu \mathrm{M}$ SU6656, an unrelated selective c-Src inhibitor (data not shown). Arrows indicate bands for the expected phosphoproteins corresponding to Fyb, Lnk, and c-Src. Results shown are representative of 3 independent experiments. The 2 panels on the right show the quantification of Western blot bands from 3 experiments (mean $\pm S D$ ). Maximal band density was defined as $100 \%$. in the presence of fibrinogen, $\mathrm{CaCl}_{2}$, and thrombin (Figure 4, D and E). Under these conditions, the clot retraction seen with $L n k^{-/-}$ platelets was slower and less effective than that seen with WT platelets, which is consistent with the idea that platelet responses are dependent on $\alpha \operatorname{IIb} \beta 3$-mediated actin cytoskeletal signaling and that Lnk is involved. To further study the role of $\alpha \operatorname{IIb} \beta 3$ activation in $L n k^{-/-}$platelets, we also used flow cytometry to quantify the specific binding of Alexa Fluor 488-conjugated fibrinogen to washed platelets upon stimulation with various concentrations of ADP, epinephrine, PAR4 peptide, phorbol myristate acetate, or convulxin (CVX), which selectively stimulates GPVI (Supplemental Figure 4A). We found that $L n k^{-/-}$and WT platelets showed similar fibrinogen binding, irrespective of the agonist inducing the inside-out signaling. In addition, levels of P-selectin expression were indistinguishable (Supplemental Figure 4B), suggesting that Lnk is not involved in $\alpha$-granule secretion.

Lnk recruitment to the $\alpha I I b \beta 3$-based signaling complex is dependent on outside-in signaling and c-Src activation. To understand the mechanism by which Lnk regulates outside-in signaling, we sought the molecule(s) that associates with Lnk in platelets. One prominent cellular event required for integrin-dependent responses is tyrosine phosphorylation of several cytosolic proteins (Figure 5A) (1, $4,27)$. Incubation of WT platelets on fibrinogen induced tyrosine phosphorylation of cellular proteins with molecular weights of 60 , 65-75, 90-110, and 120-130 kDa, but much less post-engagement tyrosine-phosphorylation was seen in $\mathrm{Lnk}^{-/-}$platelets. In addition, immunoprecipitation assays revealed that several phosphoproteins associate with Lnk (the 68-kDa protein was likely Lnk itself). A variety of proteins, including Syk, LCP2, and Fyb, are known to be tyrosine phosphorylated in an Src-dependent manner in fibrinogen-adherent platelets $(29,30)$. In the present study, both
c-Src and Fyb co-immunoprecipitated with Lnk from WT platelets adhering to fibrinogen but not from unstimulated ones; Syk was only weakly detectable, and LCP2 was barely so (Figure 5B). It has also been proposed that Fyn, an Src-family protein, may contribute to integrin $\alpha \operatorname{IIb} \beta 3$ signaling $(9,20)$, and we found that, like $\mathrm{c}-\mathrm{Src}$, Fyn associated with Lnk in stimulated platelets (Figure 5B).

c-Src and Fyn are known to associate with the cytoplasmic tail of the integrin $\beta 3$ subunit in vitro $(20,31)$, which suggests that after platelets bind to fibrinogen, Lnk regulates the assembly of an $\alpha I I b \beta 3$-based signaling complex (5). We therefore asked whether the observed association of c-Src and/or Fyb with Lnk is dependent on Src kinase activity. When platelets were incubated with $5 \mu \mathrm{M}$ PP2 (to block Src kinase activity) or PP3 (an inactive congener of PP2), PP2 but not PP3 diminished tyrosine phosphorylation of cellular proteins and the association of c-Src and Fyb with Lnk in platelets adhering to fibrinogen (Figure 5C). To then confirm that the phosphorylation of Lnk and its association with c-Src are dependent on c-Src activation, we used a COS7 cell expression system to evaluate the interaction in more detail (Supplemental Figure 5A). Flag-tagged WT Lnk (WT-Lnk) or a mutant form lacking the C-terminal portion containing Tyr536 ( $\Delta \mathrm{C}$-Lnk) was expressed in COS7 cells in the presence and absence of a constitutively active form of human c-Src (Y530F, CA-Src). Whereas WT-Lnk became tyrosine phosphorylated when coexpressed with CA-Src, $\Delta \mathrm{C}$-Lnk showed little or no phosphorylation, indicating that Tyr536 is a key target site for phosphorylation by c-Src (Supplemental Figure 5A). On the other hand, a constitutively active form of Fyn did not phosphorylate WT-Lnk (data not shown). We then evaluated the consequences of the loss of c-Src-mediated Lnk phosphorylation using $\mathrm{CHO}$ cells, which constitutively express human $\alpha \operatorname{IIb} \beta 3$ (29, 32 ) and were previously shown to spread on immobilized fibrino- 
A

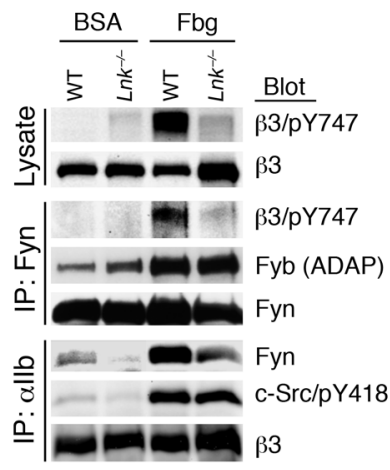

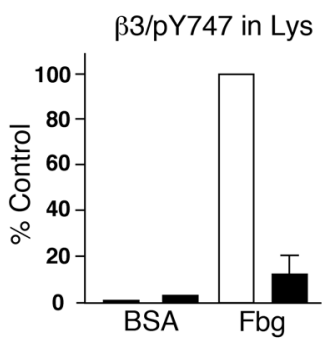

Fyn-Fyb association
Fyn-bound $\beta 3 / p Y 747$

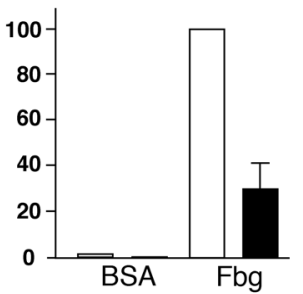

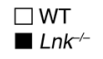

Lnk
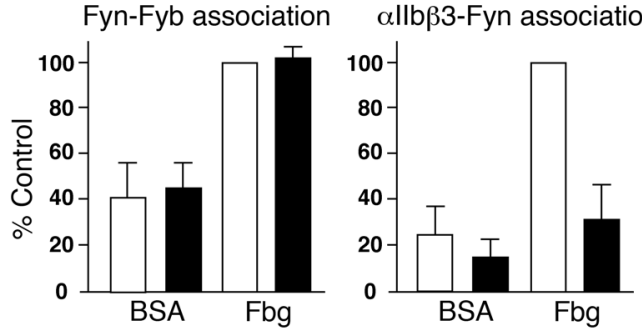

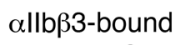

pY418 (c-Src)
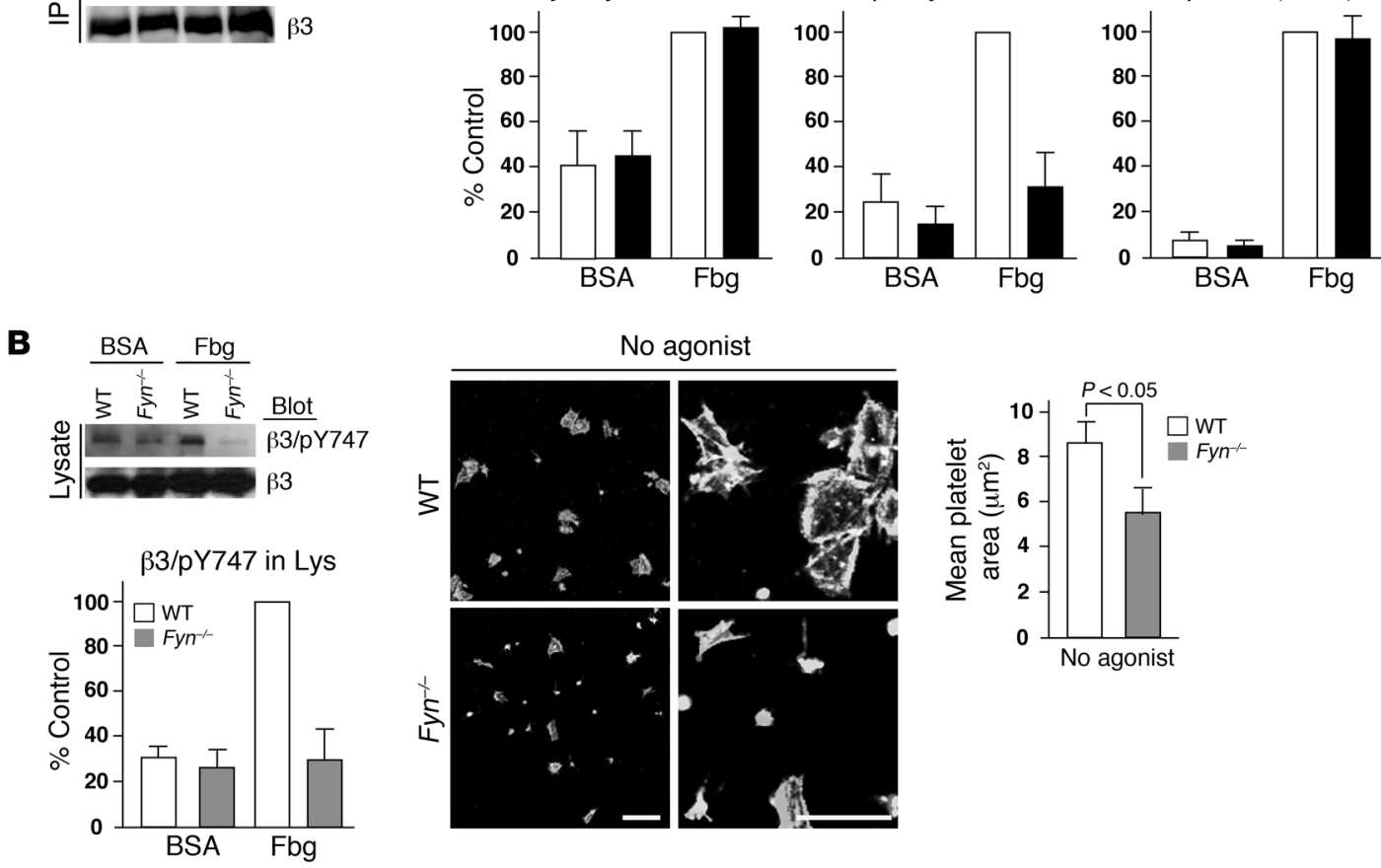

\section{Figure 6}

Lnk deficiency in platelets leads to reduced binding of Fyn to $\alpha$ llb $\beta 3$ and reduced tyrosine phosphorylation of the cytoplasmic domain of the $\beta 3$ integrin subunit. (A) WT and $L^{-1-1}$ platelets plated or maintained in suspension as in Figure 5A were lysed and analyzed by immunoblotting using anti- $\beta 3$ integrin or anti- $\beta 3 / p-T y r 747$. In other sets, immunoprecipitation was elicited with anti-Fyn or anti- $\alpha$ llb Ab. Each immunoblot panel is representative of 3 or 4 independent experiments, and estimated band densities are shown in the graphs. Band densities for WT samples from adherent platelets on fibrinogen were defined as $100 \%$. Lys, lysates. (B) Washed platelets from WT and Fyn ${ }^{-/-}$mice were plated on fibrinogen for 45 minutes or maintained in suspension in a BSA-coated dish, lysed, and analyzed. The error bars in A and B indicate mean \pm SD. (C) Left panels show the features of WT or $\mathrm{Fyn}^{-/-}$platelets allowed to spread on fibrinogen-coated coverslips for 45 minutes in the absence of agonist. The cells were fixed, permeabilized, and stained with Alexa 488 Fluor-phalloidin to visualize F-actin. Scale bars: $10 \mu \mathrm{m}$. The graph shows spreading quantified by computer analysis of their surface areas (mean $\pm \mathrm{SD}$ ).

gen in an Src kinase-dependent manner (32). When WT-Lnk or $\Delta \mathrm{C}$-Lnk was expressed as a GFP fusion protein, CHO cells expressing $\Delta C$-Lnk showed fewer lamellipodia on fibrinogen than those expressing WT-Lnk (Supplemental Figure 5B). Thus, the absence of its $\mathrm{C}$ terminus again disrupted Lnk's ability to mediate formation of lamellipodia (Supplemental Figure 5B). Apparently, the C-terminal portion of Lnk and phosphorylation of Tyr536, which is likely regulated by c-Src, are key contributors to formation of lamellipodia and cell spreading mediated via integrin $\alpha I I b \beta 3$.

Adherent Lnk $k^{-1}$ platelets show reduced tyrosine phosphorylation of the $\beta 3$ integrin subunit and reduced association of Fyn with $\alpha I I b \beta 3$. The importance of Src activation to outside-in $\alpha \mathrm{IIb} \beta 3$ signaling is well documented $(4,5,31)$. Because Lnk appears to co-immunoprecipitate with both c-Src and Fyn (Figure 5B), we next asked whether Lnk might regulate the function of these kinases in platelets. During platelet aggregation or adhesion to fibrinogen, 2 conserved tyrosine residues in the $\beta 3$ subunit, Tyr747 and Tyr759, are putatively targeted by Fyn, which is reportedly indispensable for clot retraction and prevention of re-bleeding from tail wounds (1, 8, 9). Moreover, Tyr747 is reportedly required for the binding of talin, filamin, $\mathrm{c}-\mathrm{Src}$, and other proteins essential for normal integrin signaling in platelets (33). We previously observed prominent phosphorylation of Tyr747 in WT platelets upon adherence to fibrinogen (10), but this response was severely impaired in $\mathrm{Lnk}^{-/-}$platelets (Figure 6A). Because it is likely that Fyn phosphorylates Tyr747 through direct interaction with the $\beta 3$ cytoplasmic tail $(9,20,31)$, we examined the extent to which Lnk deficiency affected (a) the association of Fyn with $\beta 3$ and (b) the phosphorylation status of Tyr747 in plate- 


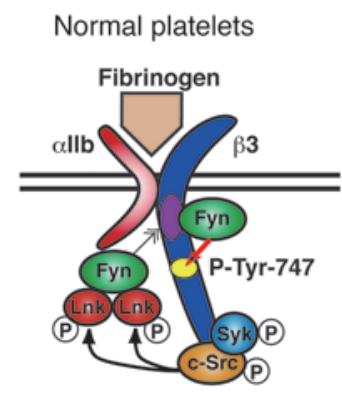

Stable thrombi

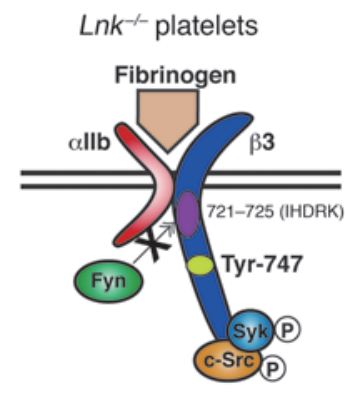

Impaired platelet spreading

Delayed clot retraction Unstable thrombi

\section{Figure 7}

Model for outside-in signaling through $\alpha \mathrm{llb} \beta 3, \mathrm{c}-\mathrm{Src}$, Fyn, and Lnk. Based on the present study, we propose that when activated, $\alpha$ llb $\beta 3$ binds to fibrinogen, and a pool of c-Src constitutively bound to the cytoplasmic domain of the integrin $\beta 3$ subunit initiates outside-in $\alpha$ llb $\beta 3$ integrin signaling, which includes phosphorylation of Syk and its recruitment to $\alpha \mathrm{llb} \beta 3$ independent of Lnk (5). The activated c-Src phosphorylates Tyr536 in a C-terminal portion of Lnk, where Lnk forms a dimeric or multimeric structure via the $\mathrm{N}$-terminal domain (34). Lnk strongly facilitates Fyn recruitment to its binding site, residues 721-725 (IHDRK) in the $\beta 3$ integrin tail, which may lead to phosphorylation of Tyr747 (P-Tyr747) within the $\beta 3$ cytoplasmic domain of integrin $\alpha \mathrm{llb} \beta 3$. Thereafter, phosphorylation of Tyr747 may facilitate thrombus stabilization in vitro and in vivo through a platelet contraction mechanism $(7,9,40)$.

lets. We found that in WT platelets, Fyn co-immunoprecipitated with phosphorylated $\beta 3$ and that this response was dependent upon fibrinogen ligation (Figure 6A, IP: Fyn). Furthermore, Fyn was bound to $\alpha \operatorname{IIb} \beta 3$ complexes immunoprecipitated 0 and 45 minutes after fibrinogen binding (Figure 6A, IP: $\alpha I I b$, lanes BSA and $\mathrm{Fbg}$, respectively), but this interaction was markedly diminished in $\mathrm{Lnk}^{-/-}$platelets at the same time point. The association of Fyn with Fyb was augmented by fibrinogen binding independently of Lnk (Figure 6A, IP: Fyn), and the activation of c-Src bound to $\alpha \operatorname{IIb} \beta 3$ (assessed based on phosphorylation of Tyr418) was comparable (Figure 6A, IP: $\alpha \mathrm{IIb}$ ). That Fyn is constitutively associated with the $\beta 3$ integrin subunit (20) suggests that Lnk contributes to the maintenance or strength of Fyn binding to the $\alpha I I b \beta 3$-based signaling complex without affecting c-Src activation.

Finally, we assessed the effect of Fyn deficiency on tyrosine phosphorylation of the $\beta 3$ subunit and the morphology of platelets spread on fibrinogen. Fyn $n^{-/-}$platelets showed reduced phosphorylation of at least Tyr747 when bound to fibrinogen for 15 or 45 minutes (shown at 45 minutes, Figure 6B). In addition, in the absence of an agonist, Fyn ${ }^{-/}$platelets exhibited delayed and impaired spreading and somewhat reduced formation of lamellipodia on fibrinogen, even after 45 minutes (Figure 6C). Fyn ${ }^{-/-}$ platelets began spreading at about 20 minutes, by which time the spreading of WT platelets had already reached a plateau (data not shown). These results are consistent with the idea that Lnk sustains Fyn kinase activation of $\alpha \operatorname{IIb} \beta 3$, thereby regulating tyrosine phosphorylation of the $\beta 3$ subunit.

Fyn-deficient mice exhibit a defect in thrombus formation similar to that seen in Lnk-deficient mice. To further examine the role of Fyn during thrombus formation in vivo, we assessed thrombus formation within mesenteric capillaries and arteries as we did for Lnk in the

experiments shown in Figure 3, C and D. We found that $\mathrm{Fyn}^{-/-}$ mice exhibited impaired thrombus formation that was very similar to that seen in Lnk $k^{-/-}$mice (Supplemental Figure 6 and Supplemental Videos 7 and 8). They also showed increased re-bleeding, but not prolonged initial bleeding times (data not shown), which is consistent with the results of Reddy et al. (20). Thus, Lnk and Fyn may act in concert to regulate $\alpha \mathrm{IIb} \beta 3$-based actin cytoskeletal reorganization leading to thrombus stabilization.

\section{Discussion}

Lnk is known to be a negative modulator of cytokine/growth factor receptor-mediated signaling (22) and to, perhaps, influence the motility of HSCs and progenitor cells, including their homing to (or lodging within) niches within the BM $(16,34)$. In addition, the proven importance of integrins and actin reorganization in HSC migration and homing $(34,35)$ and in megakaryocyte function (19) raises the possibility that Lnk modulates integrin signaling, but this idea has yet to be tested. In this report, we revealed a formerly unrecognized mechanism by which Lnk adaptor protein regulates integrin signaling in platelets and stabilizes developing thrombi in vivo. Using an in vivo $\mathrm{FeCl}_{3}$-induced vessel occlusion model and direct imaging of platelet behavior, we showed that Lnk deficiency in platelets impairs stabilization of the developing thrombus under flow conditions, which may lead to an increase in re-bleeding events in $\mathrm{Lnk}^{-/-}$(Figure 1C) and Lnk-chimeric (Supplemental Figure 2, right) mice. Through these mechanistic studies, we have been able to demonstrate that Lnk is required for platelets to fully spread on fibrinogen (Figure 4 and Supplemental Figure 3), for efficient fibrin clot retraction (Figure $4 \mathrm{C}$ ), for platelet aggregation in the presence of lower concentrations of an agonist (Figure 4D), and for thrombus stability under flow conditions in vivo (Figures 2 and 3 ). Furthermore, the findings show that all of these effects of Lnk are mediated largely by $\alpha \operatorname{IIb} \beta 3$-dependent outside-in signaling, which is likely associated with tyrosine phosphorylation of the $\beta 3$ integrin subunit (8) or Fyn tyrosine kinase (20). We also found that ligand binding to $\alpha \mathrm{IIb} \beta 3$ in platelets induces the formation of a protein complex that includes $\alpha \operatorname{IIb} \beta 3$, c-Src, Fyn, Fyb, and Lnk (Figure 5). Formation of this complex required Src kinase activity, which targeted the C-terminal portion of Lnk (Supplemental Figure 5). Finally, we showed that Lnk supports activation of Fyn within the $\alpha I I b \beta 3$ complex, that Fyn in turn tyrosine phosphorylates the $\beta 3$ subunit (Figure 6), and that the consequences of Lnk deficiency are mirrored by Fyn deficiency (Supplemental Figure 6). In contrast to the previously described constitutive association of c-Src or Fyn with $\beta 3(4,31)$, our results indicate that the efficient binding of Fyn, but not c-Src, to the $\alpha \operatorname{IIb} \beta 3$ complex requires the presence of Lnk (Figure 6). When situated in close proximity to $\alpha \operatorname{IIb} \beta 3$, Fyn may function as a positive regulator of $\beta 3$ tyrosine phosphorylation (Figure 6B) $(10,28)$, which would contribute to the stabilization of the thrombus (Supplemental Figure 6) $(9,20)$, perhaps through association of Fyn with EphA4 (7) or Bcl-3 (36). Lnk thus appears to play a previously unappreciated role in facilitating integrin $\alpha \operatorname{IIb} \beta 3$ outside-in signaling by acting in concert with Fyn to phosphorylate the $\beta 3$ subunit on Tyr747, thereby optimizing platelet cytoskeletal reorganization for stabilization of thrombi in vivo. We therefore conclude that Lnk may selectively promote Fyn kinase regulation of $\alpha \operatorname{IIb} \beta 3$ outside-in signaling in platelets.

One limitation of comparing thrombus formation in Lnk- and WT-chimeras is the need to compare platelets in mice of differ- 
ent ages due to the higher capacity for megakaryopoiesis in $L n k^{-/-}$ animals after BM transplantation. However, using intravital microscopy after laser-induced injury, we were able to compare thrombus formation in $L n k^{-/-}$and WT mice of the same age and show that thrombus formation is impaired in $L n k^{-/-}$animals despite higher numbers of circulating platelets (Figure 3, C and D). The reduced thrombus formation by Lnk-deficient platelets was even apparent 4 weeks after transplantation, at which time chimeric mice show slight thrombocytopenia, reflecting the fact that they are recovering from severe myelosuppression, and so are actively generating platelets. Furthermore, our in vitro study of the spreading of platelets from chimeric mice of different ages confirmed that the area of spread of platelets from Lnk-chimeras was consistently smaller than the area of spread of platelets from WT-chimeras. From these findings, we again conclude that Lnk deficiency leads to impaired platelet spreading independent of age or active megakaryopoiesis and contributes to the impaired stabilization of developing thrombi in vivo.

The small GTPase Rac1 and its guanosine exchange factor Vav contribute to the formation of lamellipodia during the adhesion of CHO- $\alpha$ IIb $\beta 3$ cells to fibrinogen $(29,37)$. The activation of Rac1 is mediated through 2 NxxY motifs (residues 744-747 and $756-759$ in the $\beta 3$ subunit) in the cytoplasmic tail of the $\beta 3$ subunit (37). Reddy et al. recently demonstrated that $\mathrm{CHO}$ cells expressing a truncated form of $\alpha \mathrm{IIb} \beta 3$ lacking the distal NxxY motif (residues 756-759) fail to spread on fibrinogen, and the defect is rescued by overexpression of active Fyn (20). Those results indicate that Fyn activation may augment functions of the proximal NxxY motif (residues 744-747), presumably through phosphorylation of Tyr747 (8). Our results support that idea and indicate that Fyn, with the help of Lnk, is required for Tyr747 phosphorylation of $\beta 3$ (Figure 6, A and B). In the model cell system, Lnk was directly tyrosine phosphorylated at Tyr536 by c-Src, which was required for formation of lamellipodia by $\mathrm{CHO}-\alpha \mathrm{IIb} \beta 3$ cells immobilized on fibrinogen (Supplemental Figure 5). Because Lnk supports Fyn's association with the $\alpha \operatorname{IIb} \beta 3$ complex but is not required for c-Src activation or its association with the $\alpha \operatorname{IIb} \beta 3$ complex in platelets (Figure 6A), we propose the signaling cascade depicted in Figure 7. The Fyn binding region of $\beta 3$ - residues 721-725 (IHDRK) (20) - is distinct from the c-Src binding region - residues 760-762 (RGT) (38). In normal platelets, the engagement of integrin $\alpha \operatorname{IIb} \beta 3$ initiates outside-in signaling through c-Src activation and Syk recruitment to the $\beta 3$ tail $(4,5,31)$, which is independent of Lnk. The activated c-Src presumably mediates tyrosine phosphorylation of Lnk, which leads to efficient recruitment/activation of Fyn at the $\beta 3$ tail and phosphorylation of Tyr747 (Figure 7). We studied the effects of CVX on inside-out signaling by measuring fibrinogen binding to integrin $\alpha \operatorname{IIb} \beta 3$ and on outside-in signaling by studying platelet spreading on a collagen-coated surface (data not shown). The results so far indicate that Lnk is not involved in signaling mediated through GPVI, where Fyn also functions, and it appears that Lnk deficiency does not universally affect Fyncoupled platelet receptors. Although the precise molecular mechanism is currently unknown, presumably the necessary machinery exists to ensure the specific and selective interaction of Lnk and the Fyn complex with the $\beta 3$ integrin subunit.

It has been reported that the laser-induced injury model mainly reflects the tissue factor/thrombin-mediated pathway to platelet activation and is independent of the exposed collagen-GPVI and vWF-GPIb $\alpha$ interactions triggered by the loss of endothelium; conversely, $\mathrm{FeCl}_{3}$-induced thrombus formation is collagen and $v W F$ dependent $(23,25,39)$. Nonetheless, it is reasonable to consider that our laser-induced injury model caused relatively mild damage to endothelial cells, as compared with previously reported experimental models (26). Consistent with that idea, we found that staining of the vasculature for Griffonia simplicifolia $\mathrm{IB}_{4}$ isolectin showed the endothelial layer to be intact after laser-induced injury (Supplemental Figure 7). In addition, there was no extravasation of fluorescent dyes after laser injury, indicating that vascular permeability was unaffected by laser-induced injury (Supplemental Videos 1-8). Although, as with other methods, the precise mechanism and trigger of laser-induced thrombus formation remain unclear, it is unlikely that exposure of the extracellular matrix (mainly collagen) as a result of endothelial damage is a primary trigger. One alternative possibility is that thrombus formation is triggered by ROS, but ROS produced within the blood as a result of laser irradiation of hematoporphyrin and fluorescent dyes are readily washed away by the rapid blood flow. More likely, ROS produced within endothelial cells and/or stromal spaces are involved.

Given the mild damage to the endothelium and the lack of involvement of the collagen-GPVI and vWF-GPIb $\alpha$ interactions, the diminished thrombus formation seen in $L n k^{-/-}$platelets could reflect a defect in $\alpha I I b \beta 3$-mediated signaling. Recently, 2 distinct phases in the stabilization of the primary hemostatic plug under flow conditions have been proposed. The first is a rapid phase linked to fibrin-independent platelet contractility (40), possibly accomplished through $\alpha \operatorname{IIb} \beta 3$-mediated outside-in signaling and the subsequent Rho kinase-dependent physical tightening of platelet-platelet adhesion contacts, for example, through ephrin/Eph kinases, JAM-A, or Sema4D (41). The second is a slower phase linked to thrombin generation and fibrin polymerization to stabilize the thrombus (40).

With our imaging system and laser-induced injury, we were able to observe the rapid phase of thrombus formation, which occurs within seconds, whereas $\mathrm{FeCl}_{3}$-induced arterial occlusion occurs more than 20 minutes after injury. Using chimeric mice in which platelet counts were equalized, we observed that in vivo thrombus formation by $L n k^{-/}$platelets was impaired in both models, although more significant deficiencies were detected in the laserinjury model (Figure 2C and Figure 3A).

Recent genome-wide association studies identified SNPs associated with type 1 diabetes and celiac disease, including a nonsynonymous SNP in exon 3 of LNK/SH2B3, encoding a pleckstrin homology domain (R262W) $(42,43)$. Notably, the same SNP was recently shown to be associated with increased eosinophil numbers and myocardial infarction $(42,43)$. Accordingly, it will be important to investigate the link between the sequence variation in $\mathrm{LNK} / \mathrm{SH} 2 \mathrm{~B} 3$ and platelet function in patients.

In conclusion, we explored a new regulatory mechanism by which the adaptor protein Lnk contributes to the stabilization of developing thrombi. Lnk regulates integrin $\alpha \mathrm{IIb} \beta 3$-mediated signaling in platelets and is required for Fyn interaction with $\alpha \mathrm{IIb} \beta 3$ and efficient tyrosine phosphorylation of the $\beta 3$ integrin subunit, leading to actin cytoskeletal reorganization. Because $L n k^{-/}$mice do not exhibit spontaneous (or abnormal) bleeding or severe immune system dysfunction, we suggest that molecules that modulate outside-in signaling, including Lnk, might represent novel and safe therapeutic targets for the prevention of cardiovascular events. Such therapeutics would likely pose a smaller risk for bleeding than conventional drugs. 


\section{Methods}

Cells, reagents, and mice. All reagents were from Sigma-Aldrich unless otherwise indicated. C57BL/6 mice congenic for the Ly5 locus (B6-Ly5.1) and Lnk $k^{-/}$B6-Ly5.1 mice (22) were bred and maintained at the Animal Research Centers of the Institute of Medical Science, the University of Tokyo, and of the Research Institute, International Medical Center of Japan. Lnk ${ }^{-/-}$ B6-Ly5.1 mice were first established on a C57BL/6 (B6-Ly5.2) background and backcrossed more than 12 times, after which they were crossed into the B6-Ly5.1 background. WT B6-Ly5.2 mice were purchased from Nihon SLC. $\mathrm{Fyn}^{-/-}$B6-Ly5.2 mice (44) were from T. Yamamoto (University of Tokyo). The protocol for this work was approved by the IACUCs of the Institute of Medical Science, University of Tokyo, and of the Research Institute, International Medical Center of Japan. The following Abs and reagents were used: anti-c-Src (327, a gift from J. Brugge, Harvard Medical School, Boston, Massachusetts, USA) $(4,31)$, anti-mouse $\alpha \operatorname{IIb} \beta 3$ (1B5, from B. Coller, Rockefeller University, New York, New York, USA), anti-human Lnk (from J. Hayashi, University of Maryland, Baltimore, Maryland, USA, or purchased from Abcam), anti-mouse Lnk (11), anti-Lcp2 and antiFyb (from G. Koretzky, University of Pennsylvania, Philadelphia, USA), anti-phospho-Src pY418 and anti-phospho- $\beta 3$ pY773 (Y747 in mice) (Biosource International), anti-Syk (N-19), anti-Fyn (FYN3) and anti- $\beta 3$ integrin (N-20) (Santa Cruz Biotechnology Inc.), anti-phosphotyrosine

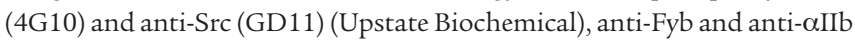
(MWReg30, BD Biosciences and Invitrogen), HRP-conjugated secondary Abs (Bio-Rad), Protein G Sepharose (GE Healthcare), protease inhibitor cocktail, aprotinin, and leupeptin (Roche Molecular Biochemicals), rhodamine-phalloidin, Alexa Fluor 488-conjugated fibrinogen, and Alexa Fluor 488-conjugated bovine IgG Abs (Molecular Probes, Invitrogen), purified human fibrinogen (American Diagnostica Inc), FITC-conjugated anti-mouse integrin $\alpha 2, \alpha I I b$, and $\beta 3$ and the FITC-Annexin-V kit (BD and Invitrogen), PE-anti-mouse GPIb $\alpha$, FITC-anti-mouse GPVI, and PE-antimouse P-selectin (Emfret), and CVX (from T. Morita, Meiji Pharmaceutical University, Tokyo, Japan).

$B M$ transplantation. BM from femurs was washed with PBS and counted. WT ( $10^{7}$ per recipient mouse) or $L^{-1} k^{-/}\left(2 \times 10^{5}\right.$ or $\left.5 \times 10^{5}\right)$ BM cells were intravenously transfused into 8 -week-old male Ly-5.2 recipient mice. The recipients were then lethally irradiated at a dose of 9.5 Gy. Two, 4, 6, and 8 weeks later, PBLs were collected from the recipient mice, and chimerism was confirmed using a Ly5.1/Ly5.2 system.

Bleeding times. Tail bleeding assays were performed with 8- to 12-week-old $\mathrm{Lnk}^{-/}$and WT male mice. Mice were anesthetized with $50 \mu \mathrm{g} / \mathrm{ml}$ pentobarbital, after which a $5-\mathrm{mm}$ segment of the distal tip of the tail was cut off, and the tail was immediately immersed in PBS at $37^{\circ} \mathrm{C}$. Tail bleeding times were defined as the time required for the bleeding to stop. Tail bleeding was monitored for at least an additional 60 seconds to detect possible re-bleeding (secondary bleeding), as previously described $(8,20,45)$. We preliminarily confirmed that tail bleeding and re-bleeding times were comparable in B6-Ly5.1 and B6-Ly5.2 mice.

Measurement of $\mathrm{FeCl}_{3}$-induced vessel occlusion times in carotid arteries. Mice were anesthetized by injection with urethane $(1.5 \mathrm{~g} / \mathrm{kg})$, and a segment of the carotid artery was exposed. Thrombus formation was then triggered by applying $10 \% \mathrm{FeCl}_{3}$ solution to the adventitial side to induce endothelial cell injury (23). FITC-dextran solution $(5 \mathrm{mg} / \mathrm{kg} \mathrm{BW}$, MW $150,000)$ was injected via the tail vein, and carotid blood flow was continuously monitored using fluorescence confocal microscopy to determine the time to occlusion.

Intravital microscopy and thrombus formation. To visually analyze thrombus formation in the microcirculation of the mesentery in living animals, we used in vivo laser injury and visualization techniques developed through modification of conventional methods $(18,24)$. Male mice were anesthe- tized by injection with urethane $(1.5 \mathrm{~g} / \mathrm{kg})$, and a small incision was made so that the mesentery could be observed without being exteriorized. FITCdextran $(5 \mathrm{mg} / \mathrm{kg} \mathrm{BW})$ was injected into mice to visualize cell dynamics, while hematoporphyrin $(1.8 \mathrm{mg} / \mathrm{kg}$ for capillary thrombi, $2.5 \mathrm{mg} / \mathrm{kg}$ for arterioles) was injected to produce ROS upon laser irradiation. Blood cell dynamics and production of thrombi were visualized during laser excitation ( $\lambda 488 \mathrm{~nm}, 30 \mathrm{~mW}$ power). Sequential images were obtained for 20 seconds at 30 frames/s using a spinning-disk confocal microscope (CSU22, Yokogawa Electronics) and an EM-CCD camera (Impactron CCD; Nihon TI). The diameters of the examined capillaries were as follows: in Figure 3A, WT-chimera $6.56 \pm 0.12 \mu \mathrm{m}$, Lnk-chimera $6.68 \pm 0.06$ $\mu \mathrm{m}(n=30$ vessels from 5 animals, $P=0.42)$; in Figure $3 \mathrm{C}, \mathrm{Lnk}^{-/-}$ $6.56 \pm 0.12 \mu \mathrm{m}$, WT $6.46 \pm 0.10 \mu \mathrm{m}$, Lnk $^{-/-} 6.56 \pm 0.12 \mu \mathrm{m}(n=30$ vessels from 5 animals, $P=0.26$ ). In Figure 3D, diameters of the examined arterioles were as follows: WT $26.6 \pm 4.5 \mu \mathrm{m}, \mathrm{Lnk}^{-/-} 25.7 \pm 3.4 \mu \mathrm{m}(n=10$ vessels from 5 animals, $P=0.43$ ). We also confirmed that thrombus formation within the vessels was comparable in the B6-Ly5.1 and B6-Ly5.2 mice.

Preparation of blood samples for analysis of platelet spreading and fibrinogen binding. For each experiment, blood samples were collected from 8-10 $\mathrm{Lnk}^{-/-}$and WT male mice (8-12 weeks old) by cardiac puncture after $\mathrm{CO}_{2}$ treatment. Some collected samples were immediately transferred to plastic tubes containing one-sixth volume of acid-citrate-dextrose (ACD). Platelet-rich plasma (PRP) was obtained by centrifugation of whole blood at $150 \mathrm{~g}$ for 15 minutes without braking. To obtain the washed platelets used for most of the in vitro experiments, $1 \mu \mathrm{M} \mathrm{PGE}_{1}$ and $2 \mathrm{U} / \mathrm{ml}$ apyrase were added, and the platelets were centrifuged at $750 \mathrm{~g}$ for 10 minutes. The sedimented platelets were then washed in modified Tyrode-HEPES buffer containing $1 \mu \mathrm{M} \mathrm{PGE}_{1}$ plus $15 \%$ volume $\mathrm{ACD}$ and finally resuspended in an appropriate volume of $\mathrm{Ca}^{2+}$-free modified Tyrode-HEPES buffer (10 mM HEPES [pH 7.4], $12 \mathrm{mM} \mathrm{NaHCO}_{3}, 138 \mathrm{mM} \mathrm{NaCl}, 5.5 \mathrm{mM}$ glucose, $2.9 \mathrm{mM} \mathrm{KCl}$, and $1 \mathrm{mM} \mathrm{MgCl}_{2}$ ).

Confocal microscopic analysis of platelet spreading and immunoprecipitation assays. All confocal studies were performed using a Leica TCS SP2 microscope equipped with a $63 \times, 1.40 \mathrm{NA}$ oil immersion objective (Leica), as described previously (46). Images were assembled using Adobe Photoshop. Analysis of platelet adhesion (cell number) and calculation of their surface area (spreading) were done using NIH ImageJ software (http:// rsbweb.nih.gov/ij/).

The lysis buffer used for immunoprecipitation contained 2\% Triton $\mathrm{X}-100$ or $1 \% \mathrm{NP}-40,150 \mathrm{mM} \mathrm{NaCl}, 50 \mathrm{mM}$ Tris- $\mathrm{HCl}$ (pH 7.4), $0.5 \mathrm{mM}$ EGTA, $0.5 \mathrm{mM}$ EDTA, $1 \mathrm{mM} \mathrm{Na}_{3} \mathrm{VO}_{4}, 0.5 \mathrm{mM} \mathrm{NaF}, 0.5 \mathrm{mM}$ PMSF, and 50 $\mu \mathrm{g} / \mathrm{ml}$ leupeptin. Anti- $\alpha \mathrm{IIb}$ was used for immunoprecipitation of $\alpha \operatorname{IIb} \beta 3$.

Flow cytometric measurement of fibrinogen-binding (activation of $\alpha I I b \beta 3$ ) and $P$-selectin expression. Washed, rested platelets were incubated for 30 minutes at room temperature with $200 \mu \mathrm{g} / \mathrm{ml}$ Alexa Fluor 488-conjugated fibrinogen plus ADP, epinephrine, PAR4 agonist peptide, or PMA in a 50- $\mu$ l final volume of modified Tyrode-HEPES buffer containing $0.2 \mathrm{mM} \mathrm{CaCl}_{2}$. The binding of Alexa Flour 488-fibrinogen to platelets was quantified using an Aria flow cytometer (BD). Nonspecific binding was determined in the presence of $20 \mu \mathrm{g} / \mathrm{ml} 1 \mathrm{~B} 5$. Specific binding was defined as total minus the nonspecific binding. P-selectin expression was measured similarly using a Canto-II flow cytometer (BD) with washed platelets in a $100-\mu \mathrm{l}$ final volume $\left(1 \times 10^{6}\right.$ platelets $)$.

Clot retraction assay. To obtain washed platelets, PRP from WT or $L n k^{-/}$ mice was diluted with 1 volume of modified Tyrode-HEPES buffer and centrifuged in the presence of $0.15 \mu \mathrm{M} \mathrm{PGE}{ }_{1}$. The sedimented platelets were then washed in modified Tyrode-HEPES buffer containing $0.15 \mu \mathrm{M}$ $\mathrm{PGE}_{1}$ and $1 \mathrm{mM}$ EDTA and finally resuspended with modified TyrodeHEPES buffer to adjust the number of platelets to $3 \times 10^{8} / \mathrm{ml}$. Fibrin clot retraction was studied by incubating the clots in the presence of $2 \mathrm{U} / \mathrm{ml}$ 
thrombin, $500 \mu \mathrm{g} / \mathrm{ml}$ fibrinogen, and $2 \mathrm{mM} \mathrm{CaCl}_{2}$ for 1 or 2 hours at $37^{\circ} \mathrm{C}$ in an aggregometer cuvette as described previously $(47,48)$.

Plasmids and transfection of COS 7 or $\mathrm{CHO}$ cells. COS7 and CHO- $\alpha \mathrm{IIb} \beta 3$ cells (provided by H. Kashiwagi, Osaka University, Osaka, Japan) were transfected using Lipofectamine 2000 methodology (Invitrogen). Constitutively active human c-Src mutant (Y530F)/pcDNA3 was obtained from T. Tezuka (University of Tokyo). Flag-tagged Lnk and the C-terminal deletion mutant ( $\Delta$ C-Lnk) were constructed by PCR using previously constructed plasmids (34) as templates and reinserted into pcDNA3 vector. A Lnk cDNA cassette lacking the stop codon was generated by PCR and inserted into pcDNADEST47 (Invitrogen) to generate the expression vector for the Lnk-GFP fusion protein, as described previously (34).

Statistics. Differences between experimental and control animals were analyzed using 2-tailed Student's $t$ tests. Incidence of re-bleeding times was evaluated by the $\chi^{2}$ test. $P$ values less than 0.05 were considered significant.

\section{Acknowledgments}

The authors thank J. Brugge, B. Coller, J. Hayashi, H. Kashiwagi, G. Koretzky, T. Morita, and T. Tezuka for providing reagents or cells and T. Yamamoto for Fyn-null mice. We are also grateful to J. Seita, H. Tsukui, A. Yamasaki, K. Wakabayashi, and M. Tajima for their excellent technical help. This work was supported by Grants-in-Aid and Special Coordination Funds for Promoting Science and Technology from the Ministry of Education, Culture, Sports, Science and Technology and from the Ministry of Health, Labour and Welfare, by the Japanese Sharyou Foundation (Tokyo, Japan), by the Uehara Memorial Foundation (Tokyo, Japan), and by the Mitsubishi Pharma Research Foundation (Osaka, Japan). H. Takizawa and S. Nishimura are supported by a fellowship for Japanese Junior Scientists from the Japan Society for the Promotion of Science.

Received for publication April 9, 2009, and accepted in revised form October 28, 2009.

Address correspondence to: Koji Eto, Division of Stem Cell Bank, The Institute of Medical Science, The University of Tokyo, 4-6-1 Shirokanedai, Minato-ku, Tokyo, 108-8639 Japan. Phone: 81-3-64092342; Fax: 81-3-6409-2343; E-mail: keto@ims.u-tokyo.ac.jp. Or to: Satoshi Takaki, Research Institute, International Medical Center of Japan, 1-21-1 Toyama, Shinjuku-ku, Tokyo, 162-8655 Japan. Phone: 81-3-3202-7181; Fax: 81-3-3208-5421; E-mail: stakaki@ri.imcj.go.jp.
1. Shattil SJ, Newman PJ. Integrins: dynamic scaffolds for adhesion and signaling in platelets. Blood. 2004;104(6):1606-1615.

2. Ruggeri ZM. Platelets in atherothrombosis. Nat Med. 2002;8(11):1227-1234.

3. Inoue $\mathrm{O}$, et al. Laminin stimulates spreading of platelets through integrin alpha6beta1-dependent activation of GPVI. Blood. 2006;107(4):1405-1412.

4. Obergfell A, et al. Coordinate interactions of Csk, Src, and Syk kinases with [alpha]IIb[beta] 3 initiate integrin signaling to the cytoskeleton. J Cell Biol. 2002;157(2):265-275.

5. Shattil SJ. Integrins and Src: dynamic duo of adhesion signaling. Trends Cell Biol. 2005;15(8):399-403.

6. Arias-Salgado EG, et al. PTP-1B is an essential positive regulator of platelet integrin signaling. $J$ Cell Biol. 2005;170(5):837-845.

7. Prevost N, et al. Eph kinases and ephrins support thrombus growth and stability by regulating integrin outside-in signaling in platelets. Proc Natl Acad Sci U S A. 2005;102(28):9820-9825.

8. Law DA, et al. Integrin cytoplasmic tyrosine motif is required for outside-in alphaIIbbeta3 signalling and platelet function. Nature. 1999;401(6755):808-811.

9. Phillips DR, Nannizzi-Alaimo L, Prasad KS. Beta3 tyrosine phosphorylation in alphaIIbbeta3 (platelet membrane GP IIb-IIIa) outside-in integrin signaling. Thromb Haemost. 2001;86(1):246-258.

10. Xi X, et al. Tyrosine phosphorylation of the integrin beta 3 subunit regulates beta 3 cleavage by calpain. J Biol Chem. 2006;281(40):29426-29430.

11. Takaki S, et al. Control of B cell production by the adaptor protein lnk. Definition of a conserved family of signal-modulating proteins. Immunity. 2000;13(5):599-609.

12. Takaki S, Morita H, Tezuka Y, Takatsu K. Enhanced hematopoiesis by hematopoietic progenitor cells lacking intracellular adaptor protein, Lnk. J Exp Med. 2002;195(2):151-160.

13. Velazquez L, et al. Cytokine signaling and hematopoietic homeostasis are disrupted in Lnk-deficient mice. J Exp Med. 2002;195(12):1599-1611.

14. Tong W, Lodish HF. Lnk inhibits Tpo-mpl signaling and Tpo-mediated megakaryocytopoiesis. J Exp Med. 2004;200(5):569-580.

15. Tong W, Zhang J, Lodish HF. Lnk inhibits erythropoiesis and Epo-dependent JAK2 activation and downstream signaling pathways. Blood. 2005;105(12):4604-4612.

16. Buza-Vidas N, et al. Cytokines regulate postna- tal hematopoietic stem cell expansion: opposing roles of thrombopoietin and LNK. Genes Dev. 2006;20(15):2018-2023.

17. Seita J, et al. Lnk negatively regulates self-renewal of hematopoietic stem cells by modifying thrombopoietin-mediated signal transduction. Proc Natl Acad Sci U S A. 2007;104(7):2349-2354.

18. Falati S, Gross P, Merrill-Skoloff G, Furie BC, Furie B. Real-time in vivo imaging of platelets, tissue factor and fibrin during arterial thrombus formation in the mouse. Nat Med. 2002;8(10):1175-1181.

19. Takizawa $H$, et al. Growth and maturation of megakaryocytes is regulated by Lnk/Sh2b3 adaptor protein through crosstalk between cytokineand integrin-mediated signals. Exp Hematol. 2008;36(7):897-906.

20. Reddy KB, Smith DM, Plow EF. Analysis of Fyn function in hemostasis and alphaIIbbeta3-integrin signaling. J Cell Sci. 2008;121(Pt 10):1641-1648.

21. Fitau J, Boulday G, Coulon F, Quillard T, Charreau B. The adaptor molecule Lnk negatively regulates tumor necrosis factor-alpha-dependent VCAM-1 expression in endothelial cells through inhibition of the ERK1 and -2 pathways. J Biol Chem. 2006;281(29):20148-20159.

22. Ema H, et al. Quantification of self-renewal capacity in single hematopoietic stem cells from normal and Lnk-deficient mice. Dev Cell. 2005;8(6):907-914.

23. Dubois C, Panicot-Dubois L, Merrill-Skoloff G, Furie B, Furie BC. Glycoprotein VI-dependent and -independent pathways of thrombus formation in vivo. Blood. 2006;107(10):3902-3906.

24. Nishimura $S$, et al. In vivo imaging in mice reveals local cell dynamics and inflammation in obese adipose tissue. J Clin Invest. 2008;118(2):710-721.

25. Dubois C, Panicot-Dubois L, Gainor JF, Furie BC, Furie B. Thrombin-initiated platelet activation in vivo is $v W F$ independent during thrombus formation in a laser injury model. J Clin Invest. 2007;117(4):953-960.

26. Furie B, Furie BC. In vivo thrombus formation. J Thromb Haemost. 2007;5(Suppl 1):12-17.

27. Juliano RL, Redding P, Alahari S, Edin M, Howe A, Aplin A. Integrin regulation of cell signaling and motility. Biochem Soc Trans. 2004;32(pt 3):443-446.

28. Blystone SD. Kinetic regulation of beta 3 integrin tyrosine phosphorylation. J Biol Chem. 2002;277(49):46886-46890.

29. Obergfell A, et al. The molecular adapter SLP76 relays signals from platelet integrin alpha-
IIbbeta3 to the actin cytoskeleton. J Biol Chem. 2001;276(8):5916-5923.

30. Jordan MS, Singer AL, Koretzky GA. Adaptors as central mediators of signal transduction in immune cells. Nat Immunol. 2003;4(2):110-116.

31. Arias-Salgado EG, et al. Src kinase activation by direct interaction with the integrin beta cytoplasmic domain. Proc Natl Acad Sci U S A. 2003; 100(23):13298-13302.

32. Salsmann A, Schaffner-Reckinger E, Kabile F, Plancon S, Kieffer N. A new functional role of the fibrinogen RGD motif as the molecular switch that selectively triggers integrin alphaIIbbeta3-dependent RhoA activation during cell spreading. J Biol Chem. 2005;280(39):33610-33619.

33. Petrich BG, et al. The antithrombotic potential of selective blockade of talin-dependent integrin alpha IIb beta 3 (platelet GPIIb-IIIa) activation. J Clin Invest. 2007;117(8):2250-2259.

34. Takizawa $\mathrm{H}$, et al. Enhanced engraftment of hematopoietic stem/progenitor cells by the transient inhibition of an adaptor protein, Lnk. Blood. 2006;107(7):2968-2975.

35. Cancelas JA, et al. Rac GTPases differentially integrate signals regulating hematopoietic stem cell localization. Nat Med. 2005;11(8):886-891.

36. Weyrich AS, et al. Signal-dependent translation of a regulatory protein, Bcl-3, in activated human platelets. Proc Natl Acad Sci U S A. 1998; 95(10):5556-5561.

37. Berrier AL, Martinez R, Bokoch GM, LaFlamme $\mathrm{SE}$. The integrin beta tail is required and sufficient to regulate adhesion signaling to Rac1. J Cell Sci. 2002;115(Pt 22):4285-4291.

38. Arias-Salgado EG, Lizano S, Shattil SJ, Ginsberg MH. Specification of the direction of adhesive signaling by the integrin beta cytoplasmic domain. J Biol Chem. 2005;280(33):29699-29707.

39. Izuhara $Y$, et al. Inhibition of plasminogen activator inhibitor-1: its mechanism and effectiveness on coagulation and fibrosis. Arterioscler Thromb Vasc Biol. 2008;28(4):672-677.

40. Ono A, et al. Identification of a fibrin-independent platelet contractile mechanism regulating primary hemostasis and thrombus growth. Blood. 2008;112(1):90-99.

41. Brass LF, Zhu L, Stalker TJ. Novel therapeutic targets at the platelet vascular interface. Arterioscler Thromb Vasc Biol. 2008;28(3):s43-s50.

42. Hunt KA, et al. Newly identified genetic risk vari- 
ants for celiac disease related to the immune response. Nat Genet. 2008;40(4):395-402.

43. Gudbjartsson DF, et al. Sequence variants affecting eosinophil numbers associate with asthma and myocardial infarction. Nat Genet. 2009;41(3):342-347.

44. Yagi T, et al. Regional localization of Fyn in adult brain; studies with mice in which fyn gene was replaced by lacZ. Oncogene. 1993;8(12):3343-3351.

45. Kasirer-Friede A, et al. ADAP is required for normal alphaIIbbeta3 activation by VWF/GP Ib-IX-V and other agonists. Blood. 2007;109(3):1018-1025.

46. Eto K, et al. The WAVE2/Abi1 complex differentially regulates megakaryocyte development and spreading: implications for platelet biogenesis and spread- ing machinery. Blood. 2007;110(10):3637-3647. 47. Prevost N, Kato H, Bodin L, Shattil SJ. Platelet integrin adhesive functions and signaling. Methods Enzymol. 2007;426:103-115.

48. Suzuki-Inoue K, et al. Involvement of Src kinases and PLCgamma2 in clot retraction. Thromb Res. 2007;120(2):251-258. 